\title{
TOOTH EXTRUSION USING FIXED-REMOVABLE APPLIANCE WITH ACRYLIC CAPPING A NEW APPROACH
}

Ghani SHA. Tooth extrusion using fixed-removable appliance acrylic capping a new approach. Annals Dent Univ. Malaya 1996; 3: 57-59

\section{ABSTRACT}

Fixed-removable appliance is frequently used to extrude a tooth but the idea of incorporating an acrylic capping or stop has not been documented in the literature. This article reports on a case treated with this new approach and describes the technique used.

Key Words: Fixed-removable, Acrylic capping , acrylic stop.

\section{Author}

Sarah H. A. Ghani

BDS, M.Sc (Med. Sci ), MDORCPS (Glasgow )

Lecturer in Orthodontics

Department of Children's Dentistry and

Orthodontics

Faculty of Dentistry

University of Malaya

59100 Kuala Lumpur.

\section{Introduction}

Fixed -removable appliance are particularly useful for the purpose of extrusion of infraoccluded or unerupted tooth (1). Directly bonded attachments such as Begg and Edgewise brackets or even composite materials are used by which a spring or an extended buccal arm can be engaged $(1,2)$. The removable appliance has an advantange of providing an excellent anchorage for vertical movement of a single tooth (3). Unlike a conventional fixed appliance, there is always the danger of intruding, tipping adjacent teeth and occlusal plane owing to reciprocal effects on the archwire (4).

This article describes a case report of tooth extrusion treated with fixed-removable appliance and an acrylic capping or stop for more controlled and guided vertical movement.

\section{Case Report}

A 15 year old female patient who had just completed an active orthodontic treatment with removable appliances in the undergraduate clinic at the Faculty of Dentistry, University of Malaya, presented with the complaint of height discrepancy of the central incisors i.e. the upper left central incisor was slightly longer than the counterpart ( Figure 1 ).

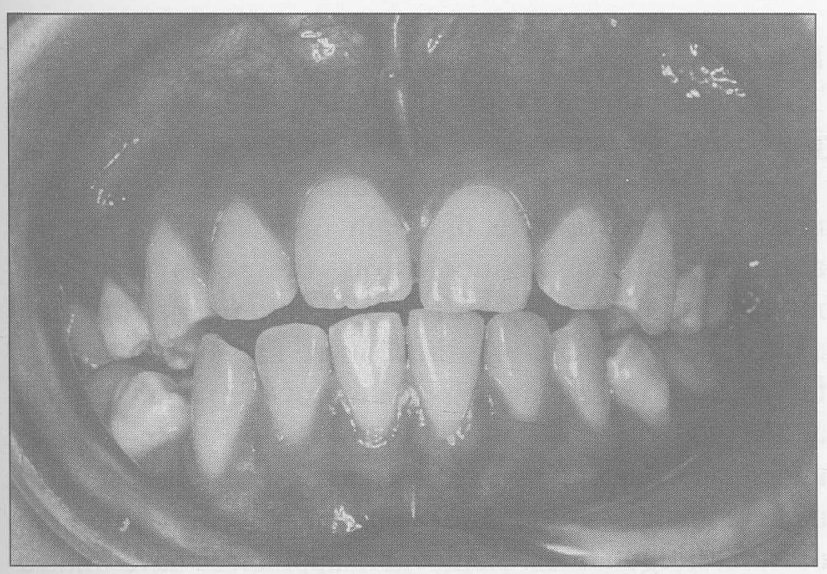

Figure 1: 15 year old female patient with the complaint of upper left central incisor longer than on the right.

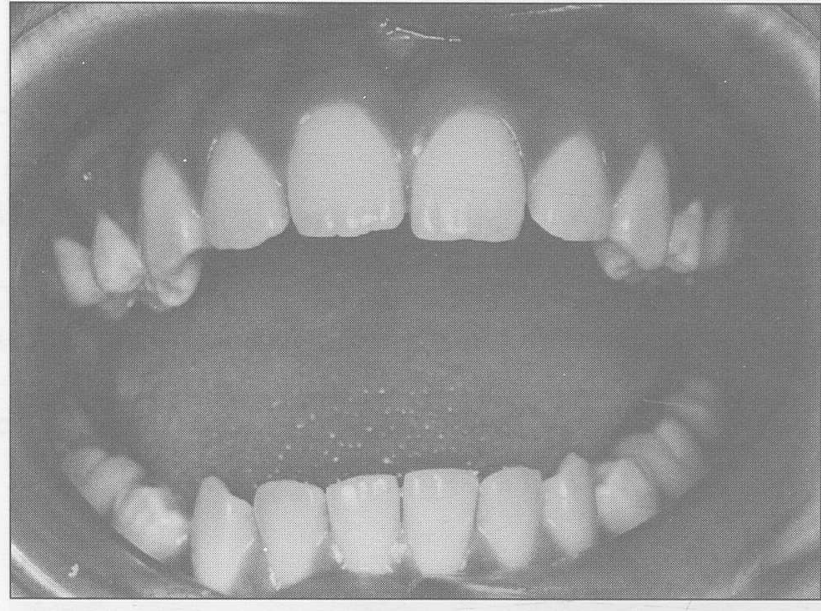

Figure 2: Intra oral examination showed that the upper right central incisor was a millimeter short of the occlusal plane and a median diastema.

The patient previously had a Class II division 1 malocclusion, an overjet of $7 \mathrm{~mm}$ with a slight overlap of the central incisors and some crowding of the lower incisors. Four first permanent bicuspids were removed.

On examination, it was noted that the problem was more of the upper right central incisor which was obviously a millimeter short of the occlusal plane (Figure 2). The patient and the parents had the impression that all anterior teeth should be of the same level. This was explained not to be so. Attention was brought to the fact that the problem was clearly the upper right central incisor rather than the left central incisor and the procedure involved to correct the levelling of the two central incisors was fully understood.

Fortunately, she also presented with a median diastema which provided sufficient space for further alignment of the incisors. With that, extrusion of the upper right central incisor was planned.

Bearing in mind that the patient was particularly aware of the slight discrepancy at the end of the 
removable appliance therapy, every effort had to be made to ensure that the tooth was not overextruded. Thus, the idea of acrylic capping or stop came to mind.

\section{The Appliance}

A working impression and a model set-up was done with the tooth in question set slightly mesial and occlusally to the level of the upper left central incisor. The removable appliance consisted of the Adams retention clasps on the upper first permanent molars and the cuspids.

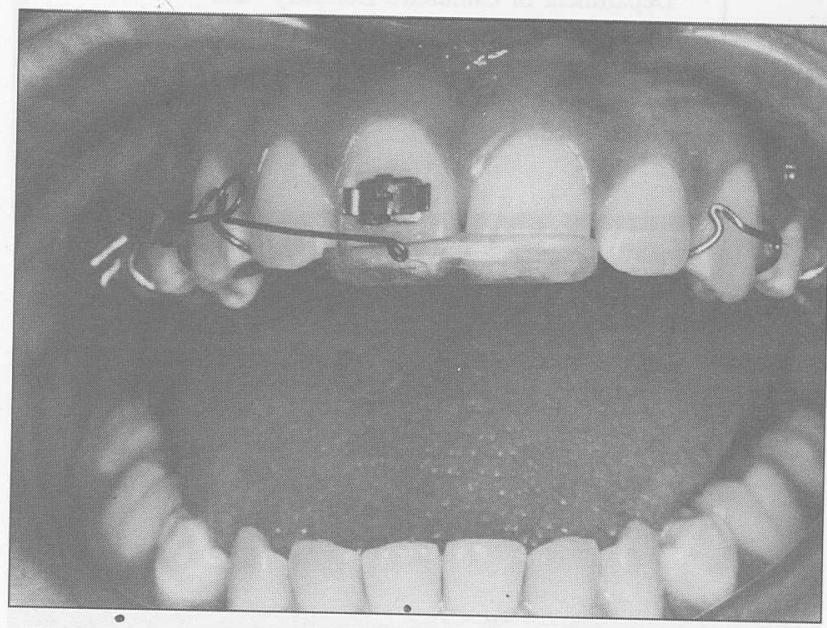

Figure 3: An extended buccal arm soldered to the bridge of the cuspid clasp and activated 1-2 mm a month.

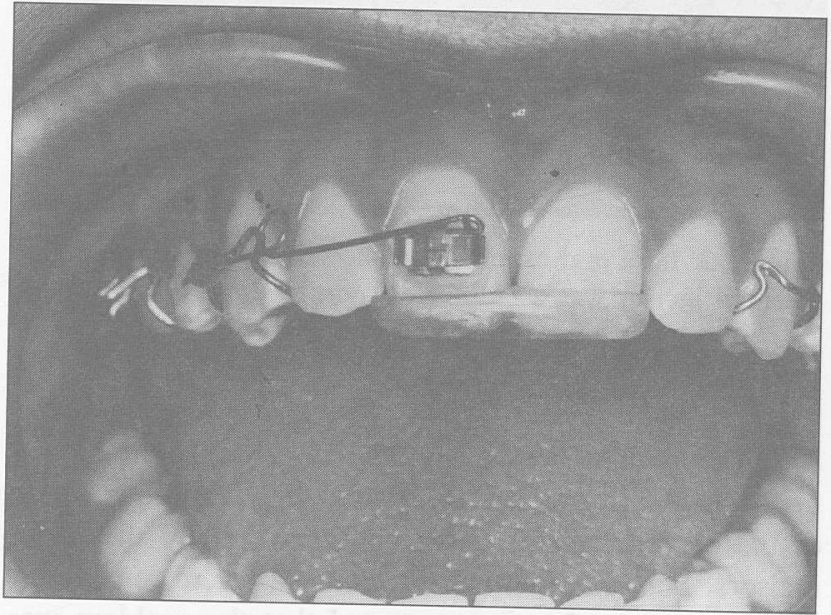

Figure 4: Instruction given regarding the wear of the appliance. Note the space between the upper right central incisor and the acrylic capping.

The acrylic baseplate was extended over the incisal edge of the pre-set central incisors. The active component of the appliance consisted of an 0.020 inch round stainless steel Australian wire with adjustment coil soldered to the bridge of the upper right cuspid clasp (Figure 3). This arm was extended anteriorly to be engaged into the slot of a Beddtiot bracket bonded onto the upper right central incisor. The extended arm was activated 1-2 mm per month and the patient received instruction of wear ( Figure 3,4).

\section{Progress}

After three months of active wear, the tooth eventually moved into the acrylic capping. The incisal edges levelled and the median diastema resolved (Figure 5). A bonded lingual retainer, 0.0175 inch multistranded wire, was used onto the palatal surfaces of the two central incisors (Figure 6).

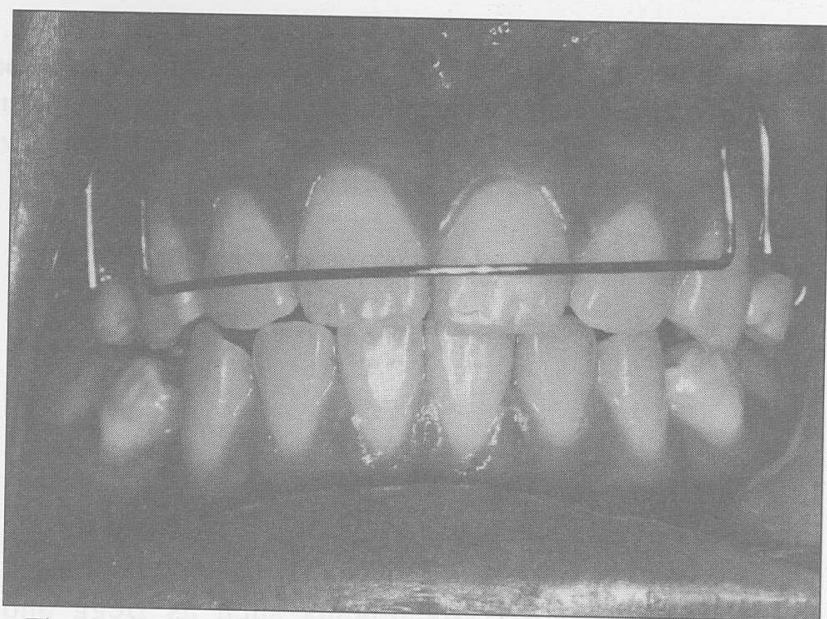

Figure 5: Upper right central incisor extruded and levelled. Median diastema closed.

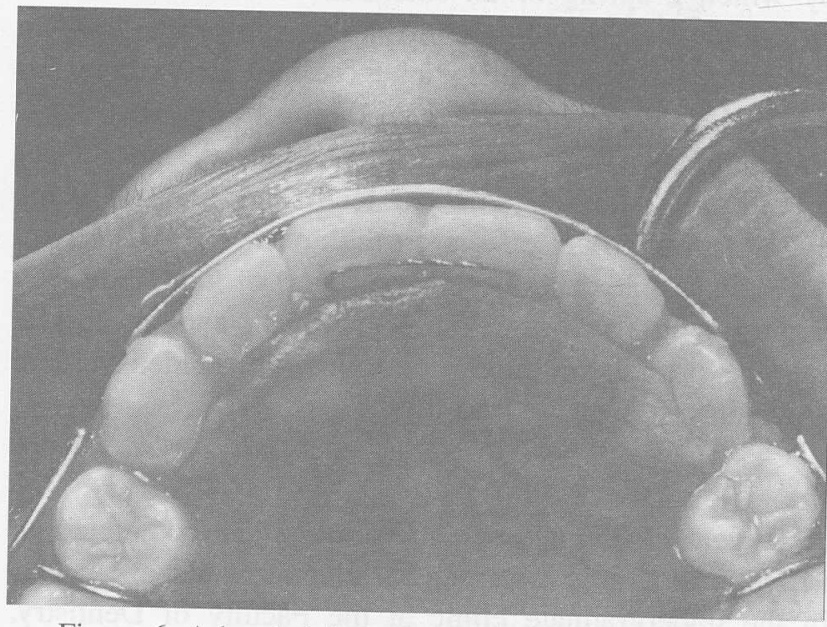

Figure 6: A bonded lingual semi-permanent retainer of 0.0175 inch multistranded wire placed.

At present, the patient is into semi-permanent full time retention reviewed regularly.

\section{Discussion}

The case presented proves that patients nowadays justifiably are expecting a higher quality of result.

This is not only technically demanding, but treatment must be based upon a thorough understanding of the aetiology of the malocclusion, the severity of the malocclusion and the different treatment options and approaches available in orthodontics. The most important factor, however, is the expectation of the patient/parents at the end of orthodontic treatment. It is important to understand that the majority of cases seen today demand more complex orthodontic therapy to fully correct the occlusal irregularities. Removable appliances have limited role in managing orthodontic cases. There are cases, 
however, where only certain obvious problems are tackled for example gross overjet or Class 1 crowding problems and accepting other minor irregularities. The patients/parents should be made fully aware of the aims of the treatment at the start and the compromised result gained using simple appliances.

The case presented would have benefited well with the use of fixed appliances but her previous records showed that the initail aim was only to reduce her overjet problem and lower arch crowding which to the author's judgement was adequately achieved. The problem of her unlevelled incisors at the end of the treatment was mainly due to the initial incisor overlap she presented at the start. As the overjet was reduced the upper right incisor which overlapped the counterpart aligned with a slight elongation. This would normally happen in any case. Perhaps if the patient was made aware earlier of the future outcome she may have opted for other treatment options or be happy to accept the result at this stage.
Thus, it is extremely important to study the case carefully and communicate well with the patients.

This approach of using fixed-removable appliance to extrude a tooth is not new. It is well documented in the literature and textbooks regarding the technique and the success of such treatment but certainly the idea of an acrylic capping or stop has yet to be reported. It is a simple approach easily accepted by the patient and adapted by the general dental practitioners. It is quite easy to overextrude a tooth on activation especially so where only a millimeter of movement is needed. The acrylic capping or stop confidently eliminates such problem and as with this particular case, one has to be extra careful or treatment could go on forever.

\section{Acknowledgement}

I would like to thank this patient for the challenge in developing this new approach.

\section{References}

1. Houston WJB, Stephen CD, Tulley WJ. Fixed appliances. In: a Textbook of Orthodontics, 2nd ed. Wright, Oxford, 1992; 319-20.

2. Houston WJB. Walter's Orthodontic Notes, Wright PSG, Bristol, 1983; 169-71.

3. Regan D. Case Report : Tooth Extrusion with a Removable Appliance. Journal of Clinical Orthodontics. 1993 ; 27: 283-4.

4. Kesling PC. Case Report : Ankylosis and the frontal occlusal plane. Journal of Clinical Orthodontics. 1991; 25: 411-3. 